\title{
Recording and Decoding of Vagal Neural Signals Related to Changes in Physiological Parameters and Biomarkers of Disease
}

\author{
Theodoros P. Zanos \\ Center for Bioelectronic Medicine, The Feinstein Institute for Medical Research, Donald \& Barbara Zucker \\ School of Medicine at Hofstra/Northwell, Manhasset, New York 11030 \\ Correspondence: tzanos@northwell.edu
}

\begin{abstract}
Our bodies have built-in neural reflexes that continuously monitor organ function and maintain physiological homeostasis. Whereas the field of bioelectronic medicine has mainly focused on the stimulation of neural circuits to treat various conditions, recent studies have started to investigate the possibility of leveraging the sensory arm of these reflexes to diagnose disease states. To accomplish this, neural signals emanating from the body's built-in biosensors and propagating through peripheral nerves must be recorded and decoded to identify the presence or levels of relevant biomarkers of disease. The process of acquiring these signals poses several technical challenges related to the neural interfaces, surgical techniques, and data-processing framework needed to record and analyze them. However, these challenges can be addressed with a rigorous experimental approach and new advances in implantable electrodes, signal processing, and machine learning methods. Outlined in this review are studies decoding vagus nerve activity as it related to inflammatory, metabolic, and cardiopulmonary biomarkers. Successfully decoding peripheral nerve activity related to disease states will not only enable the development of real-time diagnostic devices, but also help advancing truly closed-loop neuromodulation technologies.
\end{abstract}

$T^{\mathrm{h}}$ he mammalian nervous system has been shaped by evolution to control an organism's homeostasis. Different types of sensory neurons can be activated by changes in various biomarkers; temperature changes activate thermoreceptors (Schepers and Ringkamp 2010), peripheral chemoreceptors, such as glomus cells, respond to change in blood oxygenation (Kumar and Prabhakar 2012), acid-sensitive and alkalinesensitive sensory neurons are activated by changes in $\mathrm{pH}$ (Nojima et al. 2000), barorecep- tors monitor blood pressure (Schreihofer and Guyenet 2002), stretch receptors detect organ distention (Phillips and Powley 2000), and changing levels of hormones and various types of sensory neurons respond to changes in levels of metabolites. Most of the signals emanating from these peripheral sensory neurons comprise the afferent part of various autonomic reflex systems that maintain homeostasis. These circuits are activated by internal and external changes in the body's environment and initiate

Editors: Valentin A. Pavlov and Kevin J. Tracey

Additional Perspectives on Bioelectronic Medicine available at www.perspectivesinmedicine.org

Copyright (C) 2019 Cold Spring Harbor Laboratory Press; all rights reserved; doi: 10.1101/cshperspect.a034157

Cite this article as Cold Spring Harb Perspect Med 2019;9:a034157 


\section{T.P. Zanos}

the response of the motor side of these reflexes. Cellular function, metabolism, and blood flow in the visceral organs are controlled by reflexes to maintain the healthy operation of all organs. Most of the control signals responsible for the afferent and efferent arcs of these reflexes are transmitted through the vagus nerve (VN), the tenth cranial nerve. The vagus arises in the brainstem and innervates the majority of the peripheral organs (Berthoud and Neuhuber 2000). Bioelectronic medicine devices have been stimulating the $\mathrm{VN}$ at the cervical level to modulate the motor arc of various reflexes. However, owing to the fact that the $\mathrm{VN}$ is the major sensory conduit of sensory signals from peripheral organs to the brain, and roughly $80 \%$ of the fibers in the VN are sensory, it is also an ideal candidate for recording these sensory signals. This will allow us to further our understanding of the afferent arcs of these reflexes. Recording these signals from the $\mathrm{VN}$ can help identify brain-body axes. Moreover, beyond mapping peripheral circuits, deciphering this signaling will enable devices to monitor realtime disruptions in autonomic balance, potentially allowing devices to diagnose various diseases and conditions before disease symptoms arise.

Recording activity from the central nervous system has been a common approach for decades, and significant advances have been made in electrode technology, as well as surgical implantation techniques. In contrast, recording from peripheral nerves, until recently, has been focused on somatic nerves and acute or ex vivo preparations. The challenges involved in recording peripheral neural signals, especially from the VN, are related to the nerve's small size in small preclinical models, as well as the presence of the perineurium, the connective tissue that surrounds the nerves and attenuates the already weak electrical signals propagating within them. Most efforts to record these signals have been focusing on extraneural peripheral nerve recordings, surgically placing surface cuff electrodes (Loeb and Peck 1996) on nerves. These recordings have specific benefits when compared with intraneural recordings through penetrating electrodes. Their surgical placement minimizes damage (del Valle and Navarro 2013) and they have been successfully implanted in humans (Waters et al. 1985; Tan et al. 2014). However, surface recordings have a lower signal-to-noise ratio and spatial specificity (Wodlinger and Durand 2009) than recordings from electrodes inside the nerve (Micera et al. 2011; Kundu et al. 2014; McCallum et al. 2017). The signals recorded represent an aggregate of the activity of multiple nerve fibers or fascicles. Moreover, these signals are prone to various sources of noise, both physiological (cardiac, respiratory, etc.) as well as instrumentation-related. To detect, isolate, and relate this neural activity to various biomarkers, various signal-processing and machine learning methods need to be deployed.

Decades of brain research have been focused on understanding the relationship between neuronal activity and mental events, such as perception, movement, memory, emotions, and numerous other complex behaviors. This effort, commonly referred to as neural decoding, has been based on translating the language of the nervous system by establishing a quantitative representation between neural signals and behavior or cognition. Using system-identification methods and, more recently, machine learning approaches, neural decoding relates the neural signals to the outside world and interprets the neural code into concepts that we understand and care about. In the context of bioelectronic medicine, neural decoding of peripheral or central neural signals to identify disease states follows the tried and tested principles that guided decoding of brain activity. To acquire the necessary data in preclinical models, neural recordings of relevant anatomical targets need to be acquired while the system is probed using stimuli that will cause a relevant response. These stimuli could be inflammatory mediators, such as cytokines, that probe the immune reflexes, glucose or insulin that probe the metabolic system, adrenaline to cause changes in blood pressure, or lung inflation to probe the respiratory system. Using data-true, system-identification approaches, the neural recordings acquired from these experiments act as the input of the model, and the stimulus or its effect manifested as an increase or decrease of a relevant bio- 
marker acts as the output of the model. Linear or nonlinear system-identification approaches are then used to resolve the functional relationship between inputs and outputs, building a quantitative, predictive model (Marmarelis 2004; Zanos et al. 2006, 2008, 2009; Marmarelis et al. 2009).

This review attempts to outline ongoing efforts to record and decode peripheral nerve activity and relate it to physiological parameters and biomarkers of organ system function and disease. Presented in the following pages is a complete methodological framework to optimally analyze and decode $\mathrm{VN}$ activity. The first part of this review will present studies focusing on the afferent arc of the inflammatory system and efforts to relate neural signals to the presence of cytokines, mediators of inflammation (Steinberg et al. 2016; Silverman et al. 2018; Zanos et al. 2018). The second part will focus on continuing efforts to relate $\mathrm{VN}$ signals to metabolic biomarkers, specifically, blood glucose levels. The third part will summarize studies that attempt to derive blood pressure and respiratory information from $\mathrm{VN}$ recordings (Sevcencu et al. 2017, 2018). Successfully decoding $\mathrm{VN}$ activity will not only enable the development of real-time diagnostic devices for a variety of diseases and conditions, but will also inform the implementation of efficient and targeted closed-loop bioelectronic medicine devices. Knowing how the neurons in the $\mathrm{VN}$ are signaling sensory information about a disease will enable development of devices that will either stop or modify this signaling and act only when it is needed.

\section{DECODING INFLAMMATION-RELATED SENSORY NEURAL SIGNALS}

Evidence has suggested that specific neural circuits responsible for maintaining homeostasis control immune responses, mainly the inflammatory reflex (Pavlov and Tracey 2012; Chavan and Tracey 2017). The VN, which innervates the majority of an organism's peripheral organs, is the main conduit of information transfer for this reflex (Borovikova et al. 2000; Tracey 2002; Rosas-Ballina et al. 2011). Activation begins with
Decoding Vagal Neural Signals Related to Disease

infection or sterile injury leading to the release of proinflammatory mediators, such as cytokines. Two well-documented proinflammatory cytokines are tumor necrosis factor (TNF) and interleukin $1 \beta$ (IL-1 $\beta$ ). When dysregulated, these can lead to immunological impairments, as well as inflammatory conditions. These cytokines are detected in the periphery and the information is relayed through the afferent VN to the brainstem. These signals are then processed in the brain with "response signals" sent down the efferent fibers of the VN to the celiac ganglion (Rosas-Ballina et al. 2008) and subsequently to the spleen, where the release of proinflammatory cytokines is blocked (Rosas-Ballina et al. 2011). Although the efferent arc of this mechanism is well understood, little is known about the sensory afferent arc. A first step to understand this part of the mechanism is to examine the properties of peripheral neural signaling by recording the activity of the main conduit, the VN, and relate them to proinflammatory cytokines.

Recording activity from peripheral nerves presents fundamental biophysical, biological, and technological challenges (Grill et al. 2009). Past efforts to understand peripheral neural signaling related to inflammatory mediators showed that IL-1 $\beta$-induced hypothermia was mediated by signals that are carried through the VN (Watkins et al. 1995). These results have been corroborated by electrical recordings of the hepatic VN and splenic nerve in rats. IL-1 $\beta$ administration through intraportal injection caused increases in overall amplitude of the recorded activity of the VN (Niijima 1996). Moreover, recordings from the perineal, pelvic, and sacral nerve in cats were related to urinary pressure within the bladder (Bruns et al. 2011). Recent studies used VN recordings to investigate in detail the gut-to-brain circuit and its role to the microbiota (Bonaz et al. 2018), nutrient sensory transduction (Kaelberer et al. 2018), and gut-induced reward (Han et al. 2018). Adaptation of these techniques for $\mathrm{VN}$ recordings in mice is crucial because of the increased availability of genetically modified mouse strains.

Using nerve recordings, the mechanism of afferent VN signaling was further explored 
T.P. Zanos

(Steinberg et al. 2016; Silverman et al. 2018; Zanos et al. 2018) by identifying neural responses to cytokines. Prior experience in brain recordings has provided signal-processing tools to remove noise components and spurious correlations from neural signals and successfully parse out the component of the recording corresponding to actual neural activity (Zanos et al. 2011, 2012). These brain recordings, much like the nerve surface recordings, are an aggregate of the activity of multiple neurons and neuronal circuits surrounding each intracortical electrode. Using specific preprocessing steps of spike detection and classification, action potentials from individual neurons (Zanos et al. 2011, $2015,2016)$ as well as multiunit activity (Mineault et al. 2013; Bouton et al. 2016) were identified in brain recordings. The subsequent firing rates of this activity were related to motor commands (Bouton et al. 2016), oculomotor modulation of visual processing (Zanos et al. 2015, 2016), and memory tasks (Zanos et al. 2006, 2008, 2009; Marmarelis et al. 2009). Although the analysis of nerve recordings poses several different challenges than the analysis of brain recordings, using the prior art and adapting already established methods is a first step to uncover the various sources that comprise the aggregate activity recorded on the surface of nerves.

Based on the previous work described, it is reasonable to expect that cytokine neural interactions would generate specific neural signals with different spatial and temporal encoding paradigms. Recent studies developed the surgical and experimental methodology (Silverman et al. 2018), recorded cytokine-induced neural signals (Steinberg et al. 2016), and developed original algorithms used on cervical $\mathrm{VN}$ recordings to discriminate between specific cytokines TNF and IL-1 $\beta$ (Zanos et al. 2018). These studies are the first step toward developing the capability to monitor cytokine levels in real time and creating new emerging therapies for bioelectronic medicine.

The raw recording acquired from the surface of the VN (Fig. 1A,B) with the use of a cuff electrode is expected to contain physiological signals that emanate from activity propagating through its fibers. Implantation of these surface cuff electrodes requires certain key surgical and experimental steps, described in detailed in the studies that established them (Steinberg et al. 2016; Silverman et al. 2018; Zanos et al. 2018). Once implanted, the electrodes record neural signals that are low in amplitude and thus easily contaminated with nonneural signals. Sources of these signals can be physiological, such as cardiac or muscle activity. Moreover, these signals are an aggregate of propagating action potentials of fibers inside the nerve and are expected to resemble neuronal action potentials in shape and in duration. However, because individual action potentials from single fibers are not often easily detected at the surface of the nerve, the recorded neural events are most likely a result of simultaneous discharge of several fibers or groups of fibers. Traditionally, the sum of activity of a number of nerve fibers is defined as a compound action potential (CAP), in which a variable proportion of nerve fibers in a nerve trunk are discharging, either spontaneously or more frequently as a result of electrical stimulation. Thus, we will use the term CAP to refer to these detected neural events.

As mentioned previously, separating the neural activity from nonneural signals is a first step to isolate the relevant information from our recordings. Proper derivation of bipolar recordings from unipolar recordings can remove much of the nonneural noise present in the signals; however, this is not usually enough to remove all sources of noise. Recordings performed in anesthetized mice contain a minimal amount of muscle artifacts; however, cardiac-related signals are usually still present in raw recordings. Using a method of spectral decomposition or filtering, such as wavelet decomposition, the neural portion of the recording can be separated from the cardiac. An alternate way would be to record an electrocardiogram (ECG) and use the detected $\mathrm{R}$ waves as ground truth to discard cardiac spikes from the neural recordings. Subsequently, CAP waveforms can be isolated by thresholding the neural signal. Because one of the hallmarks of vagal neural recordings from anesthetized mice or rats is the respiratory modulation (Fig. 1B), which is cyclostationary, a 

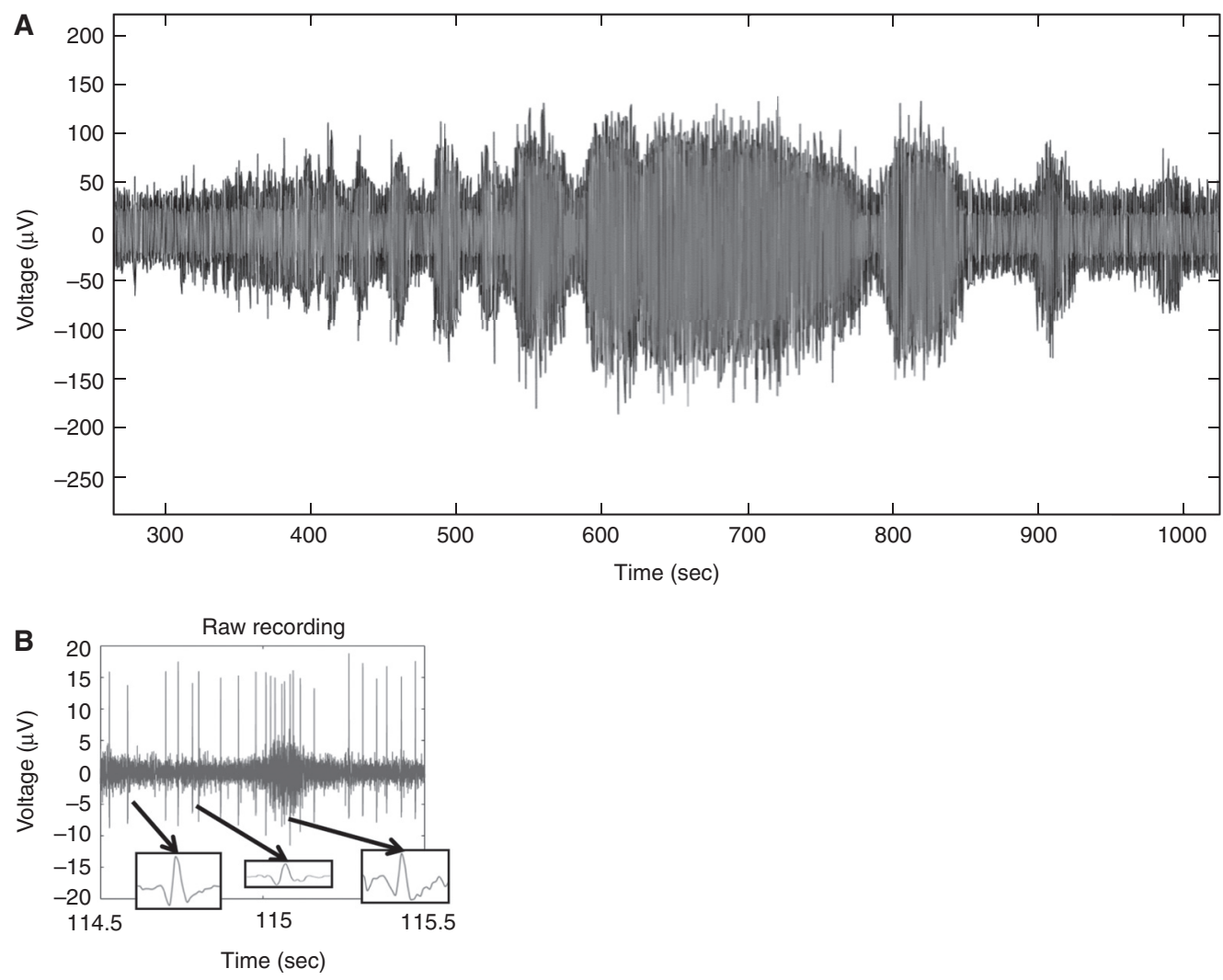

Figure 1. Trace of a raw surface bipolar nerve recording during an acute experiment. $(A)$ Lighter gray traces denote detected compound action potentials (CAPs). (B) A zoomed-in portion of a raw surface bipolar nerve recording, with the respiratory modulation and CAPs evident in the trace. (Figure based on data in Zanos et al. 2018.)

constant threshold across the total duration of a recording might not be the optimal strategy to detect impulses rising from the noise floor of the recording. As an alternative to a constant threshold, an adaptive thresholding method could be used to account for slower amplitude changes of the signal, either arising from respiratory-related modulations or changes in the nerve-electrode contact during the experiment (Zanos et al. 2018).

Activation of different groups of fibers will lead to different CAP waveform shapes and amplitudes (Fig. 1B). Several factors, such as the number of fibers discharging and their size, the propagation speed of these action potentials, and the location of these fibers relative to the recording electrode, are expected to affect the shape and amplitude of these impulses (Yoo and Durand 2005; Koh et al. 2017). To determine whether a detected waveform corresponds to activation of a specific group of fibers, it could be assigned to a specific group based on its shape and amplitude. A variety of spike-sorting methods can be used to this end (Quiroga et al. 2004; Zanos et al. 2018). Once these impulses are sorted to different CAP groups, inter-CAP-interval (ICI) histograms, which display the distribution of the intervals between two consecutive impulses, along with average CAP waveforms of these groups, can be plotted to verify their neural nature. Plotted on the right panel of Figure 2A are ICI histograms, as well as average CAP waveforms from a typical neural recording, where it is evident that the detected CAP clusters have 
T.P. Zanos
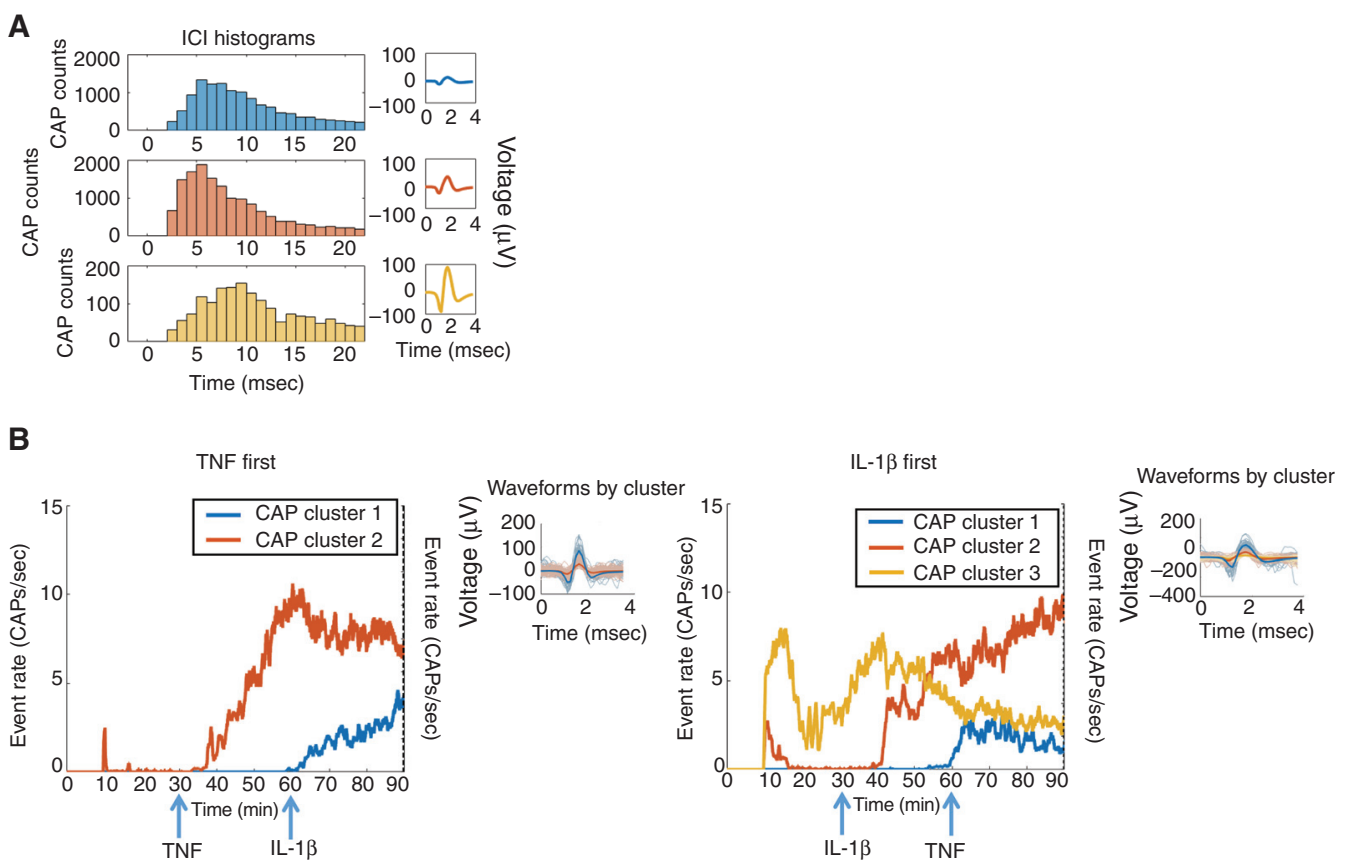

Figure 2. (A) Inter-compound action potential (CAP)-interval histograms and average CAP waveforms, as detected in a surface bipolar vagus nerve recording and clustered based on their shape and amplitude characteristics. $(B)$ Examples of neural responses to cytokines, with each colored trace representing the response rate against time of a different CAP. (Right) Subpanel for all panels includes a subset of detected CAP waveforms and the median of each CAP group in thicker lines. (Left) The vagus nerve response curve in a mouse injected first with $20 \mu \mathrm{g} / \mathrm{kg}$ tumor necrosis factor (TNF) and then with $35 \mathrm{ng} / \mathrm{kg}$ interleukin (IL)- $1 \beta$ and right panel shows the response in a mouse with the opposite injection sequence-IL-1 $\beta$ first and TNF second. (Figure based on data in Zanos et al. 2018.)

different amplitudes and firing rate characteristics. Once each waveform is assigned to a specific CAP cluster, the neural responses of these CAP groups to various stimuli can be assessed by computing their instantaneous firing rates in CAPs per second.

It has been established that TNF, IL-1 $1 \beta$, and other cytokines specifically activate calcium channels and action potentials in sensory neurons in the nodose and the dorsal root ganglion (Sorkin et al. 1997; Copray et al. 2001; Jin and Gereau 2006; Binshtok et al. 2008; Miller et al. 2009; Gudes et al. 2015). More recently (Steinberg et al. 2016; Zanos et al. 2018), to identify whether groups of neurons fire in response to TNF and IL-1 $\beta$ exposure, VN activity was recorded in mice. These studies showed that specific groups of neurons changed their firing rates after TNF injection. Two indicative examples of neural responses to the two cytokine injections are plotted in Figure 2B, with TNF injected first (left panel) and IL-1 injected first (right panel). As evident from the figure, these responses increased, plateaued, and persisted through the remainder of the recording, with subsequent IL- $1 \beta$ injection in the same animals not affecting the TNF-mediated response, but instead triggering a response from a different group of neurons. To control for the surgical and physiological manipulation caused by placing the cuff electrode and injecting $200 \mu \mathrm{L}$ of liquid, saline injection experiments consisting of two $200 \mu \mathrm{L}$ injections were performed and the $\mathrm{VN}$ response was recorded. In these experiments, the saline injections did not produce $\mathrm{VN}$ responses different than the baseline activity. 
Because a major goal of these efforts is to decode the exposure or presence of different cytokines in an organism in real time, a critical step in this analysis is to build an algorithm able to discriminate different cytokine injections by decoding $\mathrm{VN}$ responses (Fig. 3). To determine whether the cytokine-specific neural responses outlined above could be used to infer changes in levels of these cytokines, this study (Zanos et al. 2018) used a neural decoding algorithm based on the naïve Bayes method, although various other machine learning-based classification algorithms could be used to this end. The inputs to the algorithm are the distributions of firing rates of the different CAP groups, and the algorithm attempts to predict
Decoding Vagal Neural Signals Related to Disease

whether a specific firing rate value belongs to the baseline condition where no injection occurred, the post-TNF injection or the postIL-1 injection condition. It is important for these algorithms to train and test on different parts of the data to avoid overfitting and ensure generalization. Two-thirds of the data were used to train the algorithm, and the validity of the prediction was tested on the remaining one-third of these data (out-of-sample). This process was repeated until the out-of-sample data spanned all of the data and the prediction accuracy of the algorithm was quantified. When deployed, the algorithm successfully identified the three different conditions for the cytokine injection experiments; whereas, as expected, it performed poorly on sa-

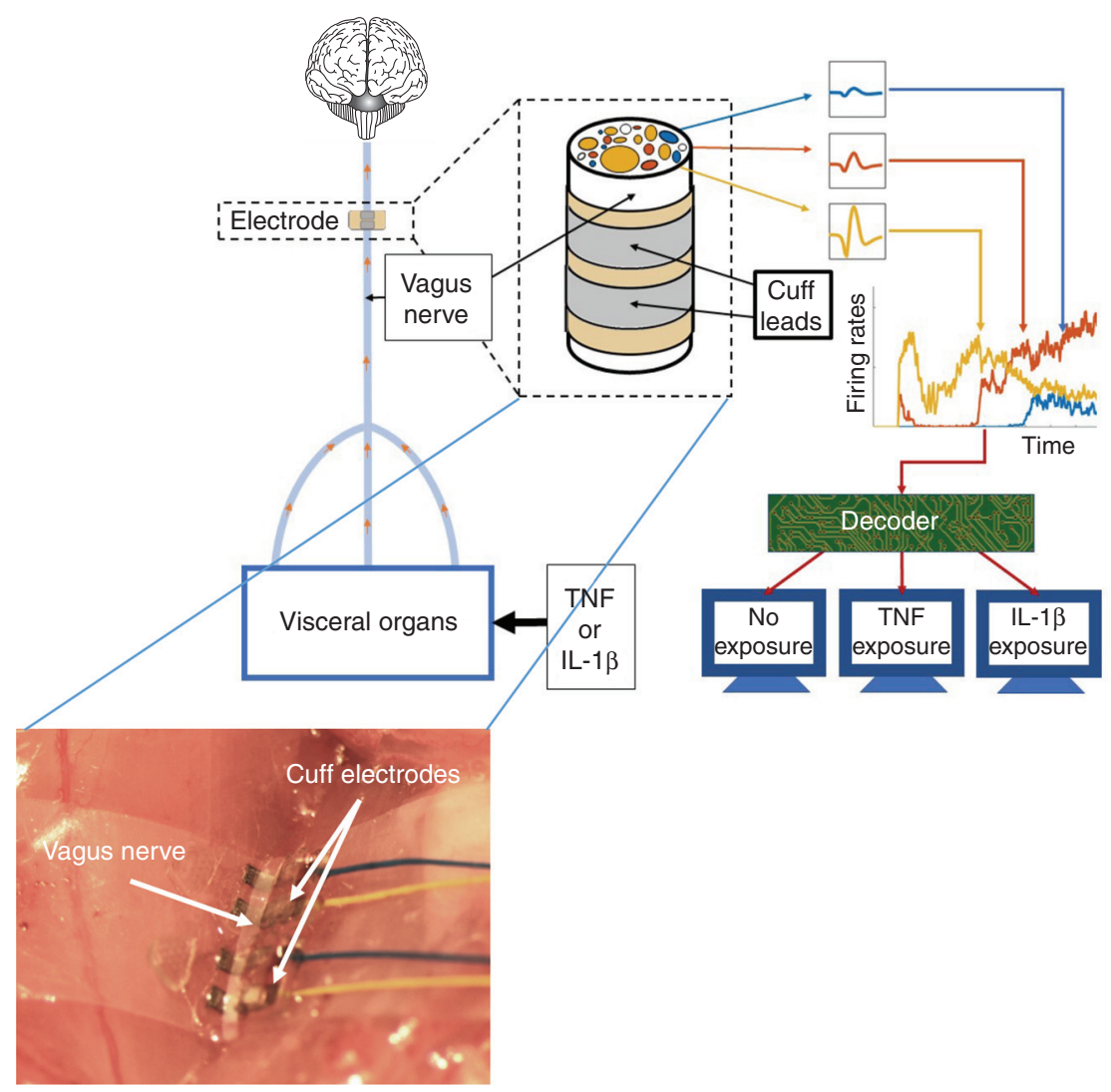

Figure 3. Schematic of the Zanos et al. (2018) methodology. Electrodes were placed on the surface of the vagus nerves of mice (left). The animals were injected with the cytokines tumor necrosis factor (TNF) and interleukin $1 \beta$ (IL-1 $\beta$ ). A decoding system (right) used a naïve Bayes machine learning algorithm to analyze data from the electrode, determining information about the mice's exposure to the cytokines. A picture of the Cortec cuff electrodes used to record vagus nerve surface potentials is shown in the bottom left panel. 
T.P. Zanos

line injection experiments. To quantify the decoding algorithm's predictive accuracy across experiments, the true positive fractions (\% correct) of all out-of-sample predictions were calculated and the population decoding success was, on average, across all injections and conditions, $83 \%$ correct, with a chance level of 33\%.

Further experiments in this study showed that $\mathrm{VN}$ recordings in IL- $1 \beta$ receptor knockout (KO) mice contain signals that only encode changes in TNF and not IL-1 levels. These results identified an IL-1R-specific signaling pathway as well as verified the decoders' ability to correctly discriminate cytokine-specific signals. Moreover, the study showed that TRPV1 $1^{+}$neurons are required to mediate cytokine responses and identified TRPV1 as a target for inflammatory signaling. By using a TRPV1 cell-depletion model and recording the $\mathrm{VN}$ activity in response to IL- $1 \beta$, no change in VN activity post-IL-1 $\beta$ administration was observed, thereby identifying TRPV $1^{+}$cell markers as required in the fibers that carry the IL- $1 \beta$ signal through the VN. Finally, administration of different doses of cytokines evoked different changes in the overall response rate of CAPs (Steinberg et al. 2016) and different firing patterns (Zanos et al. 2018), suggesting that these signals could be used to not only decode the presence but also the levels of these cytokines.

\section{SENSORY VAGAL NEURAL SIGNALS RELATED TO METABOLIC STATES}

The nervous system, primarily the brain, has long been implicated as one of the main mediators of glucose homeostasis. As the largest user of glucose, it stands to reason that the brain is involved in the processes that ensure glucose is available when needed. Past studies have focused on central mechanisms for sensing glucose and the metabolic state of an organism (Schwartz and Porte 2005); however, it has been shown that the peripheral nervous system is also involved in both sensing and relaying this information to the brain, as well as delivering the necessary motor commands to maintain glucose homeostasis (Verberne et al. 2014; Masi et al. 2018). Multiple studies have shown that hypo- thalamic sensing of metabolically important hormones such as insulin and leptin plays an important role in maintaining metabolic homeostasis (Obici et al. 2002; Schwartz et al. 2013; Inoue 2016). Because the nodose ganglion, the inferior ganglion of the cervical $\mathrm{VN}$, connects to brainstem nuclei and through that to the hypothalamus, inputs from the peripheral nervous system are reasoned to modulate hypothalamic signaling (Niijima 1989, 1998; Jordan et al. 2010). Thus, the VN would be an ideal candidate to study as it is a major conduit for the sensory neurons that connect the metabolically important organs to the brain.

Stimulation of the VN and its branches has been examined as a way to modulate blood glucose levels. Low frequency stimulation on the cervical VN has been shown to increase blood glucose in rats, and selective efferent and afferent stimulation on the same target has been shown to differentially affect insulin and blood glucose (Meyers et al. 2016). The efferent hepatic vagus has also been implicated in the control of hepatic glucose production (Matsuhisa et al. 2000; Kimura et al. 2016). Neurophysiological recordings from the $\mathrm{VN}$ in animals have confirmed its role in relaying sensory information about metabolic states. Afferent fibers of the hepatic branch of the $\mathrm{VN}$ have been shown to respond to portal vein glucose injections in a dose-dependent manner (Niijima 1984). The activity of vagal afferent neurons has been shown to be anticorrelated with intestinal glucose in type 2 diabetic rats (Lee et al. 2012) and modulated through glucosesensitive intestinal cells in the gut (Fournel et al. 2016). Much like the sensory arm of the inflammatory reflex described in the previous section of this review, this afferent nerve signaling may be part of a metabolic reflex, because hepatic nerve signals have been linked to efferent vagus-mediated responses in the pancreas (Lee and Miller 1985). Although these studies show that the VN carries important signals from the periphery to the central nervous system regarding glucose homeostasis, the nature and mechanism of these signals is still poorly understood.

Using the same neurophysiology and surgical techniques with the studies described above (Silverman et al. 2018; Zanos et al. 2018), efforts 
have been made to determine whether cervical VN activity correlates with circulating blood glucose levels in a mouse model. Surface activity from the cervical VN of mice was recorded while simultaneously measuring blood glucose levels during acute hypo- and hyperglycemia. Blood glucose levels were measured at regular time intervals (every $5 \mathrm{~min}$ before injection and every $2.5 \mathrm{~min}$ after injection). CAPs were detected using the previously described preprocessing framework (Zanos et al. 2018). The vast majority of these recordings showed a change in the firing rate of these CAPs after injecting either insulin or glucose (83.3\% for both cases). After insulin injections, these changes manifested mainly (in $80 \%$ of insulin injection experiments) as an increase in the firing rate of CAPs (Fig. 4A). Conversely, after glucose injections, these firing rates decreased (Fig. 4B, 60\% of glucose injection experiments). In control experiments of saline injections, no discernible change in the firing rate of any of the detected CAPs was observed.

Although these results show that the $\mathrm{VN}$ responds to acute hypoglycemia, it is not clear whether information about the actual blood glu- cose levels is also relayed through the same conduit. To examine this, the firing rates of CAPs were regressed to the measured blood glucose levels by performing linear regression with regularization (Fig. 5A). The ability of the regression model to estimate blood glucose levels from neural activity was quantified by computing the average error of the estimation relative to the measured blood glucose. The estimated blood glucose levels from our regression model have values close to the measured blood glucose levels (Fig. 5B), as quantified by the average error metric in all insulin injection experiments (an average error of $15 \mathrm{mg} / \mathrm{dL}$ ). Thus, signals from the surface of the cervical VN not only change with acute hypo- and hyperglycemia but, in some cases, can also be correlated with the actual levels of glucose in the blood.

For the first time, these results that correlate blood glucose levels to afferent vagus signaling showcase the feasibility of a neural-based glucose-measurement device. This device would be useful for better understanding the mechanism behind insulin resistance and hyperglycemia, as well as a novel long-term treatment plan for hyperglycemic patients
A

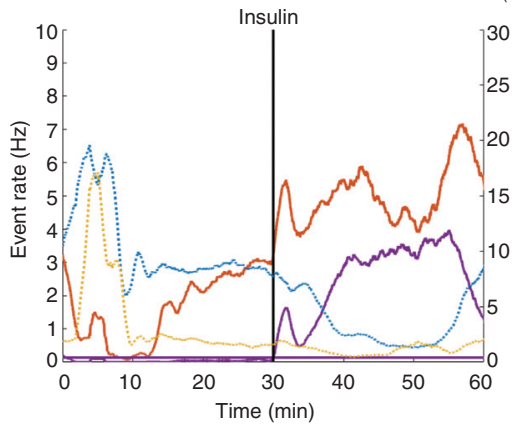

B

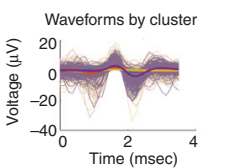

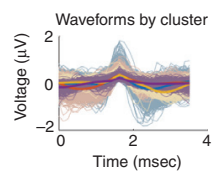

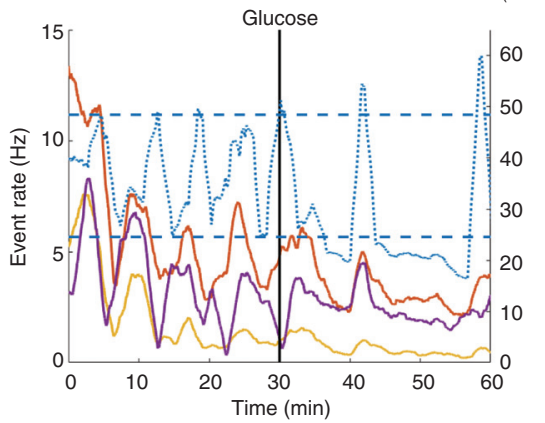

Figure 4. Examples of neural responses to different insulin and glucose injections. Each colored trace represents the response rate against time of a different compound action potential (CAP) - solid lines correspond to lower firing rate CAPs (maximum of 10-15 CAPs/sec) and dotted lines corresponds to high firing rate CAPs (maximum of 30-80 CAPs/sec). The injection is indicated by a vertical black line occurring at $30 \mathrm{~min}$. The thresholds encapsulating the baseline statistics of a particular CAP are indicated by horizontal lines of the same color. An example of $(A)$ insulin $\mathrm{VN}$ response curves that include a CAP that increases its firing rate, and $(B)$ glucose VN response curves that include a CAP that decreases its firing rate, along with their respective waveforms. 

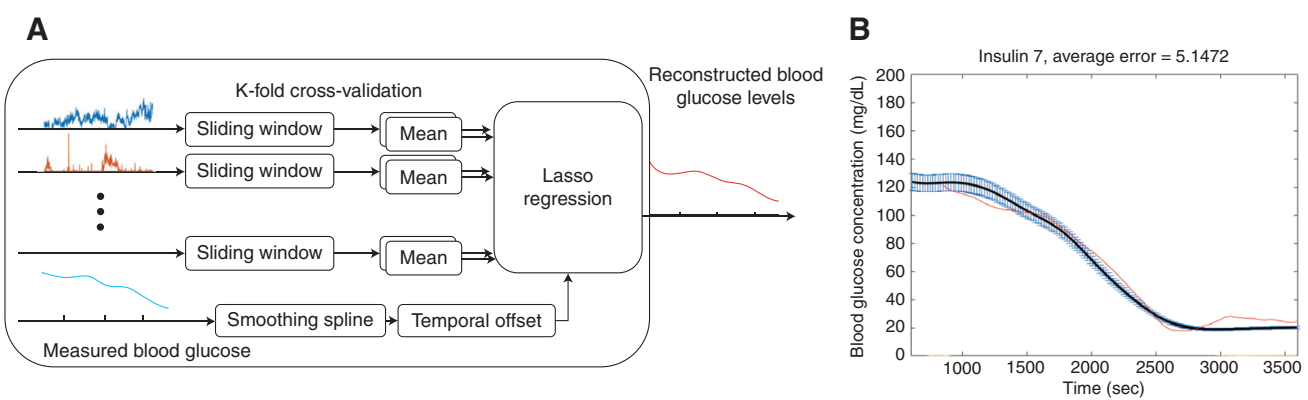

Figure 5. (A) Schematic of the decoding algorithm used to regress the compound action potential (CAP) event rates to the blood glucose concentration. The sliding-window mean of the event rates for the previous 4 min were used as features to the lasso regression model. The inputs were regressed against a non-time-shifted version of the measurements of the blood glucose concentration, using 12 -fold, leave-one-out cross-validation. (B) Example of the regression model performance in an insulin injection experiment. The black line indicates the measured blood glucose level, with blue bars indicating interpolation error. The red line indicates the estimated blood glucose levels as an output of the regression model.

\section{NEURAL BIOMARKERS OF RESPIRATION AND BLOOD PRESSURE}

Beyond inflammation and metabolic states, stimulation of the left $\mathrm{VN}$ has been a target of bioelectronic therapies to lower blood pressure in patients with resistant hypertension (Gierthmuehlen and Plachta 2016; Ng et al. 2016). In this case, a closed-loop VN stimulator driven by a blood pressure marker is essential because a device like this would only need to stimulate the nerve when hypertension occurs. However, there are conditions (e.g., exercising) when hypertension is not only normal but necessary. Thus, an ideal device for the treatment of resistant hypertension would require another biomarker or physiological signal to avoid stimulation in such conditions, for example, a respiratory marker. Because it is known that the left VN conducts respiratory-related signals (Zanos et al. 2018), a recent study investigated whether $\mathrm{VN}$ signals can be used to derive a marker to monitor respiration in anesthetized pigs (Sevcencu et al. 2018). Five of the animals were subjected to changes of their respiratory frequency and nine were subjected to changes of their respiratory volume. The left VN activity was recorded using two cuff electrodes and the respiratory cycles were monitored using a pressure transducer. The correlation between the pressure and respiratory-related neural profiles was investigated in relation to the magnitude and timing of respiratory cycles. The respiratory-related neural profiles were similar to respiratory cycles in terms of the duration and magnitude of inflation and deflation components of respiration (Fig. 6A). These profiles and the respiratory cycles were significantly linearly correlated across all animals. Through proximal and distal vagotomies, the afferent or efferent nature of these signals was determined, with the respiratory-related neural profiles being comprised mostly of efferent signals. Similar respiratory information was derived in the previously described study (Zanos et al. 2018) with the use of an adaptive algorithm that detected both the occurrence and duration of respiratory modulation. However, it should be noted that in both studies, the animals were anesthetized and that in awake-behaving animals respiration and the signals related to it could change dramatically. Nevertheless, these results show that respiratory-related $\mathrm{VN}$ activity could be used as a proxy for respiration cycles.

Another biomarker that would be essential for closed-loop VN stimulators that treat resistant hypertension would be a blood pressurerelated neural signal marker. A recent study investigated whether such a biomarker could be identified by recording neural signals using a tripolar VN implant (Sevcencu et al. 2017). Seventeen pigs were anaesthetized and implanted 
A

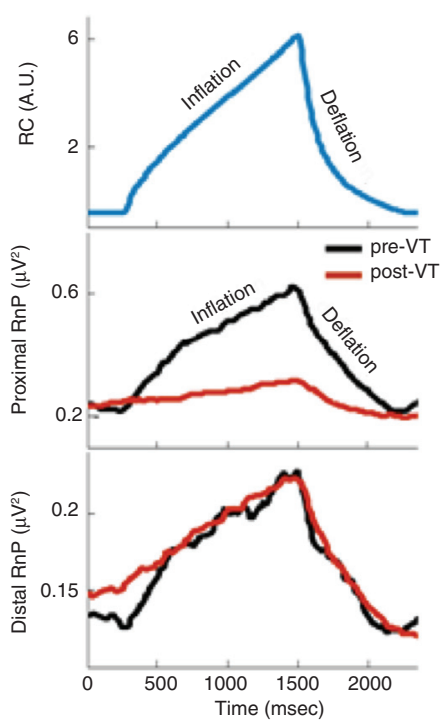

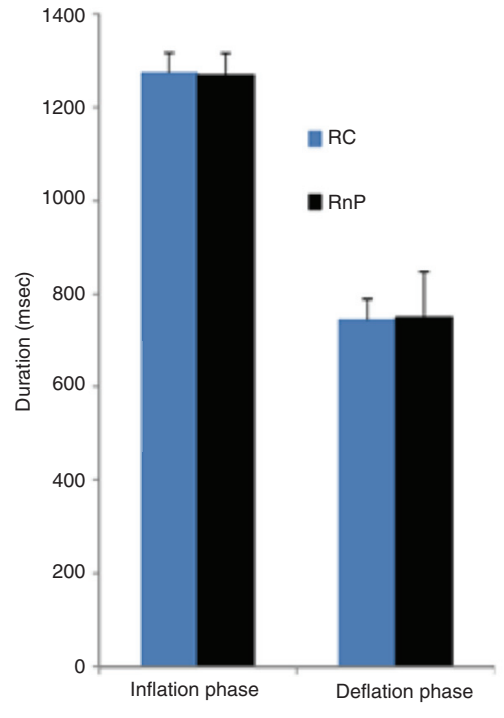

B
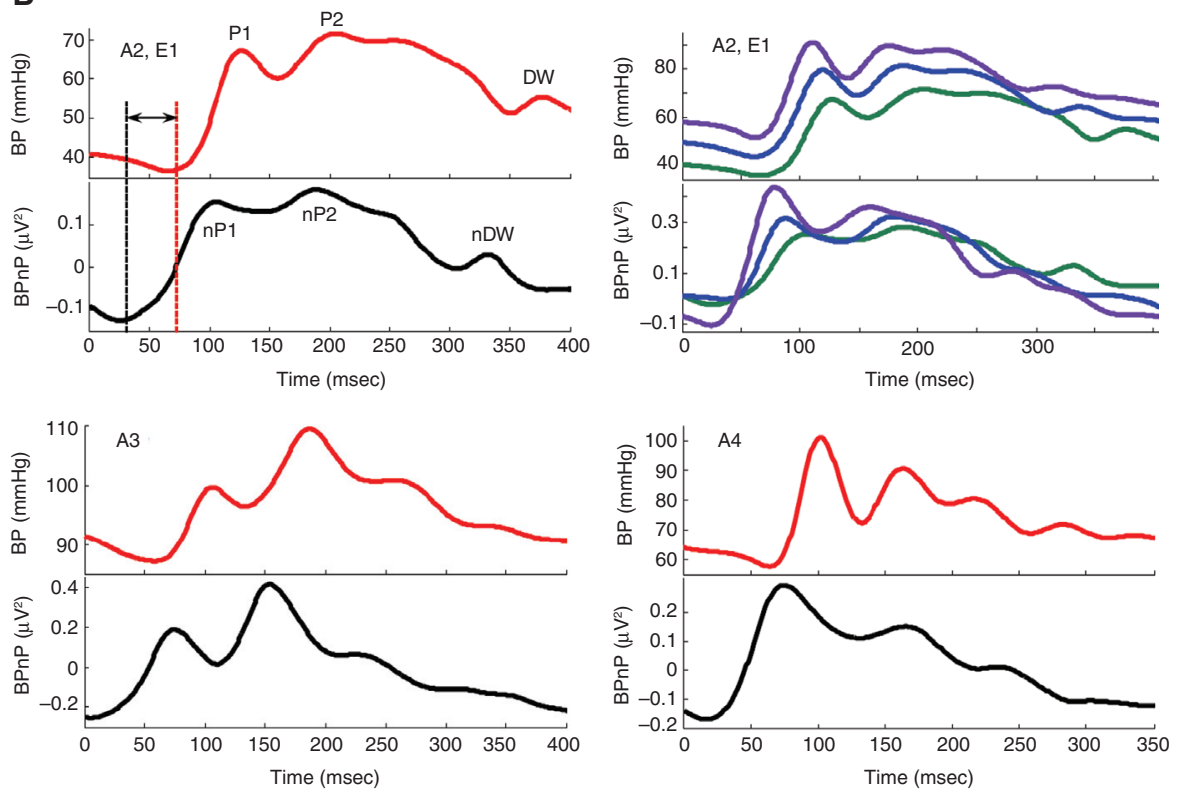

Figure 6. (A) Respiratory neural profiles recorded from the left vagus nerve were morphologically similar to respiratory cycles in terms of the duration and magnitude of inflation and deflation components of respiration and the magnitude and timing of these profiles were not correlated in the postproximal vagotomy experiments, implying an efferent nature. (Panel based on data in Sevcencu et al. 2018.) (B) Averaging blood pressure neural profiles and blood pressure signals revealed that they were morphologically identical with a lag between the two signals. The first two systolic peaks $(\mathrm{P} 1, \mathrm{P} 2)$ and dicrotic waves $(\mathrm{nP} 1, \mathrm{nP} 2$, and $\mathrm{nDW}$ ) were present in the $\mathrm{BP}$ signals and neural signals of animal 2 (first injection: top left panel, second injection: top right panel), animal 3 (bottom left panel) and animal 4 (bottom right panel). (Panel based on data in Sevcencu et al. 2017.) 


\section{T.P. Zanos}

with two cuff electrodes on the left VN (5-7 cm distance between electrodes). Arterial blood pressure was measured by implanting a catheter in the right carotid artery. Following a 5-min baseline period, a transient blood pressure increase was induced by intravenous administration of adrenaline. In ten pigs, the blood pressure levels were increased to three consecutive levels and maintained for a brief period. ECG signals were also recorded during the period of experiment. The neural signals and blood pressure signals were ensemble averaged relative to the R-peak of ECG. Squaring the raw VN signals provided the blood pressure-related neural profile. Averaging the neural-related and the blood pressure signals revealed that they were morphologically identical with a lag between the two signals (Fig. 6B). The authors speculated that this phase delay $(\sim 40 \mathrm{msec})$ between the signals was mostly likely because of the $8-10$ $\mathrm{cm}$ separation between the carotid artery pressure catheter and VN cuff electrode. Across all animals and blood pressure increment paradigms (one level of blood pressure increase or three consecutive levels of blood pressure increase), the systolic, diastolic, and mean blood pressures were linearly correlated to their respective neural profiles. Thus, VN activity and the blood pressure-related potentials could potentially serve as a blood pressure marker in implantable systems.

\section{CONCLUDING REMARKS}

The studies presented above and their promising results provide the first crucial step in understanding the sensory afferent arc of peripheral neural response circuits related to inflammatory, metabolic, pulmonary, and cardiac function. To further elucidate the nature and the spatiotemporal patterns of this signaling as they relate to homeostatic changes, as well as to develop algorithms and devices that will diagnose disease-related changes in real time, a significant amount of multidisciplinary studies will be needed. Microfabrication of peripheral nerve electrodes with higher density, biocompatibility and stability, chronically implanted recording and stimulation devices, as well as novel minia- turized implantable sensors of relevant biomarkers are key technologies that will enable further discoveries. Moreover, further improvement in signal processing and machine learning frameworks, developing more robust to noise and more computationally compact (thus ported easily to an implantable microcircuit) algorithms are crucial steps toward building real-time diagnostic and monitoring devices.

Multiple factors can modulate VN activity, including digestion, cardiac, and respiratory function, as well as infection and injury. Elements of acute experiments can also affect the recorded signals, such as level of anesthetic, body temperature, experiment duration, and electrode placement. In most of the studies outlined above, all these factors were carefully controlled and standardized to establish consistency as well as to remove nonrelated biological signals. Because of the VN's innervation of the gut, animals needed to be fasted to minimize digestion-related signaling. High levels of anesthesia can blunt neural activity, thus anesthetic levels need to be maintained at a low enough dose that allows neural responses, while keeping the animal properly anesthetized.

Future work will build on this foundation by identifying vagal signals in chronically implanted animals allowing for recordings to be performed in normal biological conditions, in which both the efficacy and the longevity of both the signals and the mapping can be assessed. This crucial next step in this line of work involves developing a mouse model with chronically implanted nerve electrodes and biosensors of various relevant biomarkers. Chronically implanted electrodes in the VN of mice (with connectors placed on the head of the animal) will be able to record the neural responses to various stimuli across multiple days of experiments and relate them directly to the various monitored biomarkers, enabling the elucidation of these relationships under normal conditions. In addition, whereas physiologically relevant neural information can be resolved with single (bipolar) surface recordings, using novel nerve interfaces that feature multiple electrodes, both extraneural and/or intraneural, could provide further insights on the nature of these signals, 
as well as improve decoding accuracy by increasing the information content captured in these signals. Moreover, optogenetic and optical imaging methods can provide unprecedented access to selectively stimulating and recording activity in these circuits. Using in vivo optical imaging and electrophysiology, neural responses of different fiber types, as captured through CAPs can be related to responses to various stimuli from different neural populations in vagal ganglia and the brainstem. Using optogenetic stimulation, the effects of selective activation and deactivation of different fiber types on the physiology of the organism can also be assessed and related to concurrent neural recordings. Last, although the mouse is a uniquely useful animal model because of the large number of genetic modifications available, it imposes some limitations. The overall low blood volume of the animal does not allow for multiple blood draws within a short period of time without changes to its biological homeostasis and its small size limits electrode designs, as well as the capabilities for chronic interfaces. Larger animal models, such as rats and pigs, could be used in parallel future studies to allow for implantation of multilead interfaces both on the vagus as well as other nerves. Moreover, they would allow multiple blood draws to monitor and correlate, in real time, neural signals to not only the exposure, but also to the actual levels of circulating inflammatory markers.

Future steps also involve the testing of chronic, closed-loop interfaces that would not only record neural signals to decode levels of biomarkers, but also modulate the signals appropriately by applying parametric nerve stimulation. Although the selection of stimulation parameters to consistently up- or down-regulate various biomarkers remains a daunting task, resolving the encoding of these biomarkers by the nervous system can provide valuable insight into the optimization of these parameters. By understanding the signals that encode changes in biomarkers, the ranges of parameter values can be narrowed down (mainly the frequency but also pulse amplitude and shape) and the dimensionality and complexity of the stimulation optimization problem can be reduced significantly.
Bioelectronic medicine combines neuroscience, molecular biology, and bioengineering to tap into the nervous system to help the body heal itself. Making effective and targeted bioelectronic medicine devices relies on how much information we have about a condition and its neural signaling. Once we know how the neurons in the $\mathrm{VN}$ are signaling the disruption of homeostasis in various diseases, we can develop devices that will diagnose this disruption in its earliest stages, even before symptoms arise. These devices would then stimulate the nerve to either stop or modify this signaling and act only when it is needed. By combining neurophysiology, neurotechnology, and machine learning and data analytics, the presented studies emphasize the importance of decoding neural signals and lay the groundwork to provide the first crucial steps toward this important goal.

\section{ACKNOWLEDGMENTS}

I thank Dr. Subash Padmanaban and Todd Levy for developing analysis tools outlined above and helping to edit this review, Samantha Kerath for diligent proofreading of this review, Dr. Harold Silverman, Dr. Emily Battinelli Masi, and Dr. Téa Tsaava for offering their impeccable surgical skills to acquire these unique data and help editing this review, and Dr. Kevin Tracey, Chad Bouton, and Dr. Yousef Al-Abed for their leadership and guidance.

\section{REFERENCES}

Berthoud HR, Neuhuber WL. 2000. Functional and chemical anatomy of the afferent vagal system. Auton Neurosci 85: 1-17. doi:10.1016/S1566-0702(00)00215-0

Binshtok AM, Wang H, Zimmermann K, Amaya F, Vardeh D, Shi L, Brenner GJ, Ji RR, Bean BP, Woolf CJ, et al. 2008. Nociceptors are interleukin-1 $\beta$ sensors. J Neurosci 28: 14062-14073. doi:10.1523/jneurosci.3795-08.2008

Bonaz B, Bazin T, Pellissier S. 2018. The vagus nerve at the interface of the microbiota-gut-brain axis. Front Neurosci 12: 49. doi:10.3389/fnins.2018.00049

Borovikova LV, Ivanova S, Zhang M, Yang H, Botchkina GI, Watkins LR, Wang H, Abumrad N, Eaton JW, Tracey KJ. 2000. Vagus nerve stimulation attenuates the systemic inflammatory response to endotoxin. Nature 405: 458462. doi: $10.1038 / 35013070$

Bouton CE, Shaikhouni A, Annetta NV, Bockbrader MA, Friedenberg DA, Nielson DM, Sharma G, Sederberg PB, 
T.P. Zanos

Glenn BC, Mysiw WJ, et al. 2016. Restoring cortical control of functional movement in a human with quadriplegia. Nature 533: 247-250. doi:10.1038/nature17435

Bruns TM, Gaunt RA, Weber DJ. 2011. Estimating bladder pressure from sacral dorsal root ganglia recordings. Conf Proc IEEE Eng Med Biol Soc 2011: 4239-4242.

Chavan SS, Tracey KJ. 2017. Essential neuroscience in immunology. J Immunol 198: 3389-3397. doi:10.4049/jim munol.1601613

Copray JCVM, Mantingh I, Brouwer N, Biber K, Küst BM, Liem RSB, Huitinga I, Tilders FJH, Van Dam AM, Boddeke HWGM. 2001. Expression of interleukin-1 $\beta$ in rat dorsal root ganglia. J Neuroimmunol 118: 203-211. doi:10.1016/S0165-5728(01)00324-1

del Valle J, Navarro X. 2013. Interfaces with the peripheral nerve for the control of neuroprostheses. Int Rev Neurobiol 109: 63-83. doi:10.1016/B978-0-12-4200456.00002-X

Fournel A, Marlin A, Abot A, Pasquio C, Cirillo C, Cani PD, Knauf C. 2016. Glucosensing in the gastrointestinal tract: Impact on glucose metabolism. Am J Physiol Gastrointest Liver Physiol 310: G645-G658. doi:10.1152/ajpgi. 00015.2016

Gierthmuehlen M, Plachta DTT. 2016. Effect of selective vagal nerve stimulation on blood pressure, heart rate and respiratory rate in rats under metoprolol medication. Hypertens Res 39: 79-87. doi:10.1038/hr.2015.122

Grill WM, Norman SE, Bellamkonda RV. 2009. Implanted neural interfaces: Biochallenges and engineered solutions. Annu Rev Biomed Eng 11: 1-24. doi:10.1146/annurevbioeng-061008-124927

Gudes S, Barkai O, Caspi Y, Katz B, Lev S, Binshtok AM. 2015. The role of slow and persistent TTX-resistant sodium currents in acute tumor necrosis factor- $\alpha$-mediated increase in nociceptors excitability. $J$ Neurophysiol 113: 601-619. doi:10.1152/jn.00652.2014

Han W, Tellez LA, Perkins MH, Perez IO, Qu T, Ferreira J, Ferreira TL, Quinn D, Liu ZW, Gao XB, et al. 2018. A neural circuit for gut-induced reward. Cell 175: 665-678. e23. doi:10.1016/j.cell.2018.08.049

Inoue H. 2016. Central insulin-mediated regulation of hepatic glucose production [review]. Endocr J 63: 1-7. doi:10.1507/endocrj.EJ15-0540

Jin X, Gereau RW. 2006. Acute p38-mediated modulation of tetrodotoxin-resistant sodium channels in mouse sensory neurons by tumor necrosis factor- $\alpha$. J Neurosci 26: 246255. doi:10.1523/jneurosci.3858-05.2006

Jordan SD, Könner AC, Brüning JC. 2010. Sensing the fuels: Glucose and lipid signaling in the CNS controlling energy homeostasis. Cell Mol Life Sci 67: 3255-3273. doi: 10.1007/s00018-010-0414-7

Kaelberer MM, Buchanan KL, Klein ME, Barth BB, Montoya MM, Shen X, Bohórquez DV. 2018. A gut-brain neural circuit for nutrient sensory transduction. Science 361: eaat5236. doi:10.1126/science.aat5236

Kimura K, Tanida M, Nagata N, Inaba Y, Watanabe H, Nagashimada M, Ota T, Asahara S, Kido Y, Matsumoto M, et al. 2016. Central insulin action activates Kupffer cells by suppressing hepatic vagal activation via the nicotinic $\alpha 7$ acetylcholine receptor. Cell Rep 14: 2362-2374. doi:10.1016/j.celrep.2016.02.032
Koh RG, Nachman AI, Zariffa J. 2017. Use of spatiotemporal templates for pathway discrimination in peripheral nerve recordings: A simulation study. J Neural Eng 14: 016013. doi:10.1088/1741-2552/14/1/016013

Kumar P, Prabhakar NR. 2012. Peripheral chemoreceptors: Function and plasticity of the carotid body. Compr Physiol 2: 141-219.

Kundu A, Wirenfeldt M, Harreby KR, Jensen W. 2014. Biosafety assessment of an intra-neural electrode (TIME) following sub-chronic implantation in the median nerve of Göttingen minipigs. Int J Artif Organs 37: 466-476. doi:10.5301/ijao.5000342

Lee KC, Miller RE. 1985. The hepatic vagus nerve and the neural regulation of insulin secretion. Endocrinology 117: 307-314. doi:10.1210/endo-117-1-307

Lee J, Cummings BP, Martin E, Sharp JW, Graham JL, Stanhope KL, Havel PJ, Raybould HE. 2012. Glucose sensing by gut endocrine cells and activation of the vagal afferent pathway is impaired in a rodent model of type 2 diabetes mellitus. Am J Physiol Regul Integr Comp Physiol 302: R657-R666. doi:10.1152/ajpregu.00345.2011

Loeb GE, Peck RA. 1996. Cuff electrodes for chronic stimulation and recording of peripheral nerve activity. $J \mathrm{Neu}$ rosci Methods 64: 95-103. doi:10.1016/0165-0270(95) 00123-9

Marmarelis PVZ. 2004. Nonlinear dynamic modeling of physiological systems. Wiley, Hoboken, NJ.

Marmarelis VZ, Zanos TP, Berger TW. 2009. Boolean modeling of neural systems with point-process inputs and outputs. Part I: Theory and simulations. Ann Biomed Eng 37: 1654-1667. doi:10.1007/s10439-009-9736-8

Masi EB, Valdés-Ferrer SI, Steinberg BE. 2018. The vagus neurometabolic interface and clinical disease. Int J Obes 42: 1101-1111. doi:10.1038/s41366-018-0086-1

Matsuhisa M, Yamasaki Y, Shiba Y, Nakahara I, Kuroda A, Tomita T, Iida M, Ikeda M, Kajimoto Y, Kubota M, et al. 2000. Important role of the hepatic vagus nerve in glucose uptake and production by the liver. Metabolism 49: 1116. doi:10.1016/S0026-0495(00)90538-9

McCallum GA, Sui X, Qiu C, Marmerstein J, Zheng Y, Eggers TE, Hu C, Dai L, Durand DM. 2017. Chronic interfacing with the autonomic nervous system using carbon nanotube (CNT) yarn electrodes. Sci Rep 7: 11723. doi:10.1038/s41598-017-10639-w

Meyers EE, Kronemberger A, Lira V, Rahmouni K, Stauss HM. 2016. Contrasting effects of afferent and efferent vagal nerve stimulation on insulin secretion and blood glucose regulation. Physiol Rep 4: e12718. doi:10.14814/ phy2.12718

Micera S, Rossini PM, Rigosa J, Citi L, Carpaneto J, Raspopovic S, Tombini M, Cipriani C, Assenza G, Carrozza $\mathrm{MC}$, et al. 2011. Decoding of grasping information from neural signals recorded using peripheral intrafascicular interfaces. J Neuroeng Rehabil 8: 53. doi:10.1186/17430003-8-53

Miller RJ, Jung H, Bhangoo SK, White FA. 2009. Cytokine and chemokine regulation of sensory neuron function. Handb Exp Pharmacol 194: 417-449. doi:10.1007/9783-540-79090-7_12

Mineault PJ, Zanos TP, Pack CC. 2013. Local field potentials reflect multiple spatial scales in V4. Front Comput Neurosci 7: 21. doi:10.3389/fncom.2013.00021 
Ng FL, Saxena M, Mahfoud F, Pathak A, Lobo MD. 2016 Device-based therapy for hypertension. Curr Hypertens Rep 18: 61. doi:10.1007/s11906-016-0670-5

Niijima A. 1984. Reflex control of the autonomic nervous system activity from the glucose sensors in the liver in normal and midpontine-transected animals. J Auton Nerv Syst 10: 279-285. doi:10.1016/0165-1838(84) 90025-0

Niijima A. 1989. Neural mechanisms in the control of blood glucose concentration. J Nutr 119: 833-840. doi:10.1093/ jn/119.6.833

Niijima A. 1996. The afferent discharges from sensors for interleukin $1 \beta$ in the hepatoportal system in the anesthetized rat. J Auton Nerv Syst 61: 287-291. doi:10.1016/ S0165-1838(96)00098-7

Niijima A. 1998. Afferent signals from leptin sensors in the white adipose tissue of the epididymis, and their reflex effect in the rat. J Auton Nerv Syst 73: 19-25. doi:10.1016/ S0165-1838(98)00109-X

Nojima K, Sumii K, Sumii M, Okahara S, Haruma K, Yoshihara M, Kajiyama G. 2000. Acid-sensitive and alkalinesensitive sensory neurons regulate $\mathrm{pH}$ dependent gastrin secretion in rat. Dig Dis Sci 45: 1217-1226. doi:10.1023/ A:1005570507166

Obici S, Zhang BB, Karkanias G, Rossetti L. 2002. Hypothalamic insulin signaling is required for inhibition of glucose production. Nat Med 8: 1376-1382. doi:10.1038/ nm1202-798

Pavlov VA, Tracey KJ. 2012. The vagus nerve and the inflammatory reflex-Linking immunity and metabolism. Nat Rev Endocrinol 8: 743-754. doi:10.1038/nrendo.2012.189

Phillips RJ, Powley TL. 2000. Tension and stretch receptors in gastrointestinal smooth muscle: Re-evaluating vagal mechanoreceptor electrophysiology. Brain Res Rev 34: 1-26. doi:10.1016/S0165-0173(00)00036-9

Quiroga RQ, Nadasdy Z, Ben-Shaul Y. 2004. Unsupervised spike detection and sorting with wavelets and superparamagnetic clustering. Neural Comput 16: 1661-1687. doi:10.1162/089976604774201631

Rosas-Ballina M, Ochani M, Parrish WR, Ochani K, Harris YT, Huston JM, Chavan S, Tracey KJ. 2008. Splenic nerve is required for cholinergic antiinflammatory pathway control of TNF in endotoxemia. Proc Natl Acad Sci 105: 11008-11013. doi:10.1073/pnas.0803237105

Rosas-Ballina M, Olofsson PS, Ochani M, Valdés-Ferrer SI, Levine YA, Reardon C, Tusche MW, Pavlov VA, Andersson U, Chavan S, et al. 2011. Acetylcholine-synthesizing $\mathrm{T}$ cells relay neural signals in a vagus nerve circuit. Science 334: 98-101. doi:10.1126/science.1209985

Schepers RJ, Ringkamp M. 2010. Thermoreceptors and thermosensitive afferents. Neurosci Biobehav Rev 34: 177184. doi:10.1016/j.neubiorev.2009.10.003

Schreihofer AM, Guyenet PG. 2002. The baroreflex and beyond: Control of sympathetic vasomotor tone by gabaergic neurons in the ventrolateral medulla. Clin Exp Pharmacol Physiol 29: 514-521. doi:10.1046/j.14401681.2002.03665.x

Schwartz MW, Porte D. 2005. Diabetes, obesity, and the brain. Science 307: 375-379. doi:10.1126/science.1104344

Schwartz MW, Seeley RJ, Tschöp MH, Woods SC, Morton GJ, Myers MG, D'Alessio D. 2013. Cooperation between brain and islet in glucose homeostasis and diabetes. $\mathrm{Na}$ ture 503: 59-66. doi:10.1038/nature12709

Sevcencu C, Nielsen TN, Struijk JJ. 2017. A neural blood pressure marker for bioelectronic medicines for treatment of hypertension. Biosens Bioelectron 98: 1-6. doi:10.1016/ j.bios.2017.06.031

Sevcencu C, Nielsen TN, Kjaergaard B, Struijk JJ. 2018. A respiratory marker derived from left vagus nerve signals recorded with implantable cuff electrodes. Neuromodulation 21: 269-275. doi:10.1111/ner.12630

Silverman HA, Stiegler A, Tsaava T, Newman J, Steinberg BE, Masi EB, Robbiati S, Bouton C, Huerta PT, Chavan SS, et al. 2018. Standardization of methods to record vagus nerve activity in mice. Bioelectron Med 4: 3 . doi:10.1186/s42234-018-0002-y

Sorkin LS, Xiao W-H, Wagner R, Myers RR. 1997. Tumour necrosis factor- $\alpha$ induces ectopic activity in nociceptive primary afferent fibres. Neuroscience 81: 255-262. doi:10.1016/S0306-4522(97)00147-4

Steinberg BE, Silverman HA, Robbiati S, Gunasekaran MK, Tsaava T, Battinelli E, Stiegler A, Bouton CE, Chavan SS, Tracey KJ, et al. 2016. Cytokine-specific neurograms in the sensory vagus nerve. Bioelectron Med 2016: 7-17.

Tan DW, Schiefer MA, Keith MW, Anderson JR, Tyler J, Tyler DJ. 2014. A neural interface provides long-term stable natural touch perception. Sci Transl Med 6: 257ra138. doi:10.1126/scitranslmed.3008669

Tracey KJ. 2002. The inflammatory reflex. Nature 420: 853859. doi:10.1038/nature 01321

Verberne T, Sabetghadam A, Korim W. 2014. Neural pathways that control the glucose counterregulatory response. Front Neurosci 8: 38. doi:10.3389/fnins.2014.00038

Waters RL, McNeal DR, Faloon W, Clifford B. 1985. Functional electrical stimulation of the peroneal nerve for hemiplegia. Long-term clinical follow-up. J Bone Jt Surg Am 67: 792-793. doi:10.2106/00004623-19856705000016

Watkins LR, Goehler LE, Relton JK, Tartaglia N, Silbert L, Martin D, Maier SF. 1995. Blockade of interleukin-1 induced hyperthermia by subdiaphragmatic vagotomy: Evidence for vagal mediation of immune-brain communication. Neurosci Lett 183: 27-31. doi:10.1016/03043940(94)11105-R

Wodlinger B, Durand DM. 2009. Localization and recovery of peripheral neural sources with beamforming algorithms. IEEE Trans Neural Syst Rehabil Eng 17: 461468. doi:10.1109/TNSRE.2009.2034072

Yoo PB, Durand DM. 2005. Selective recording of the canine hypoglossal nerve using a multicontact flat interface nerve electrode. IEEE Trans Biomed Eng 52: 1461-1469. doi:10.1109/TBME.2005.851482

Zanos TP, Courellis SH, Hampson RE, Deadwyler S, Marmarelis VZ, Berger TW. 2006. A multi-input modeling approach to quantify hippocampal nonlinear dynamic transformations. In 28th Annual International Conference of the IEEE Engineering in Medicine and Biology Society, 2006. EMBS'06, pp. 4967-4970.

Zanos TP, Courellis SH, Berger TW, Hampson RE, Deadwyler S, Marmarelis VZ. 2008. Nonlinear modeling of causal interrelationships in neuronal ensembles. IEEE Trans Neural Syst Rehabil Eng 16: 336-352. doi:10.1109/ TNSRE.2008.926716 
T.P. Zanos

Zanos TP, Hampson RE, Deadwyler SE, Berger TW, Marmarelis VZ. 2009. Boolean modeling of neural systems with point-process inputs and outputs. Part II: Application to the rat hippocampus. Ann Biomed Eng 37: 16681682. doi:10.1007/s10439-009-9716-Z

Zanos TP, Mineault PJ, Pack CC. 2011. Removal of spurious correlations between spikes and local field potentials. J Neurophysiol 105: 474-486. doi:10.1152/jn. 00642.2010

Zanos S, Zanos TP, Marmarelis VZ, Ojemann GA, Fetz EE. 2012. Relationships between spike-free local field potentials and spike timing in human temporal cortex. J Neurophysiol 107: 1808-1821. doi:10.1152/jn.00663.2011
Zanos TP, Mineault PJ, Nasiotis KT, Guitton D, Pack CC. 2015. A sensorimotor role for traveling waves in primate visual cortex. Neuron 85: 615-627. doi:10.1016/j.neuron. 2014.12.043

Zanos TP, Mineault PJ, Guitton D, Pack CC. 2016. Mechanisms of saccadic suppression in primate cortical area V4. J Neurosci 36: 9227-9239. doi:10.1523/jneurosci.101516.2016

Zanos TP, Silverman HA, Levy T, Tsaava T, Battinelli E, Lorraine PW, Ashe JM, Chavan SS, Tracey KJ, Bouton CE. 2018. Identification of cytokine-specific sensory neural signals by decoding murine vagus nerve activity. Proc Natl Acad Sci 2017: 19083. 


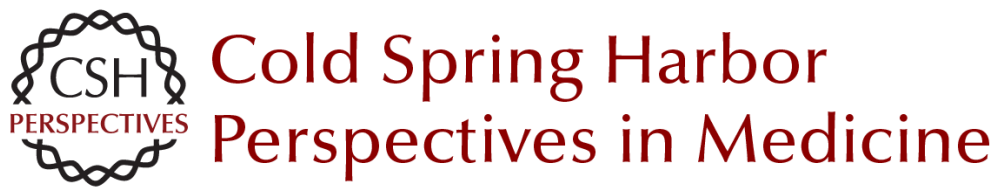

\title{
Recording and Decoding of Vagal Neural Signals Related to Changes in Physiological Parameters and Biomarkers of Disease
}

\author{
Theodoros P. Zanos \\ Cold Spring Harb Perspect Med 2019; doi: 10.1101/cshperspect.a034157 originally published online \\ January 22, 2019
}

\section{Subject Collection Bioelectronic Medicine}

Neural Control of Inflammation: Bioelectronic

Medicine in Treatment of Chronic Inflammatory

Disease

Michael Eberhardson, Laura Tarnawski, Monica

Centa, et al.

Noninvasive Neuromodulation of Peripheral Nerve Pathways Using Ultrasound and Its Current

Therapeutic Implications

Christopher Puleo and Victoria Cotero

Enteric Neuromodulation for the Gut and Beyond Yogi A. Patel and Pankaj J. Pasricha

Optogenetic Control of the Peripheral Nervous System

Rui B. Chang

Closed-Loop Neuromodulation in Physiological and Translational Research

Stavros Zanos

Electrical Impedance Methods in Neuromuscular Assessment: An Overview

Seward B. Rutkove and Benjamin Sanchez

Optogenetic Medicine: Synthetic Therapeutic

Solutions Precision-Guided by Light

Haifeng Ye and Martin Fussenegger

Technobiology's Enabler: The Magnetoelectric Nanoparticle

Sakhrat Khizroev
Bioelectronic Medicine: From Preclinical Studies on the Inflammatory Reflex to New Approaches in Disease Diagnosis and Treatment

Valentin A. Pavlov, Sangeeta S. Chavan and Kevin

J. Tracey

Vagus Nerve Stimulation and the Cardiovascular System

Michael J. Capilupi, Samantha M. Kerath and

Lance B. Becker

Harnessing the Inflammatory Reflex for the

Treatment of Inflammation-Mediated Diseases

Yaakov A. Levine, Michael Faltys and David Chernoff

Recording and Decoding of Vagal Neural Signals Related to Changes in Physiological Parameters and Biomarkers of Disease Theodoros P. Zanos

Restoring Movement in Paralysis with a

Bioelectronic Neural Bypass Approach: Current

State and Future Directions Chad E. Bouton

Bioelectronic Medicine--Ethical Concerns Samuel Packer, Nicholas Mercado and Anita Haridat

Use of Bioelectronics in the Gastrointestinal Tract Larry Miller, Aydin Farajidavar and Anil Vegesna

Vagus Nerve Stimulation at the Interface of BrainGut Interactions

Bruno Bonaz, Valérie Sinniger and Sonia Pellissier

For additional articles in this collection, see http://perspectivesinmedicine.cshlp.org/cgi/collection/ 